\title{
Balanced consistency and balanced cost reduction for sequencing problems
}

\author{
René van den Brink • Youngsub Chun
}

Received: 19 March 2010 / Accepted: 24 January 2011 / Published online: 2 April 2011

(C) The Author(s) 2011. This article is published with open access at Springerlink.com

\begin{abstract}
We investigate the implications of balanced consistency and balanced cost reduction in the context of sequencing problems. Balanced consistency requires that the effect on the payoff from the departure of one agent to another agent should be equal between any two agents. On the other hand, balanced cost reduction requires that if one agent leaves a problem, then the total payoffs of the remaining agents should be affected by the amount previously assigned to the leaving agent. We show that the minimal transfer rule is the only rule satisfying efficiency and Pareto indifference together with either one of our two main axioms, balanced consistency and balanced cost reduction.
\end{abstract}

\section{Introduction}

Consider a group of agents who must be served in a facility. The facility can handle only one agent at a time and agents incur waiting costs. We assume that an agent's waiting cost is constant per unit of time, but that agents differ in the unit waiting cost and the amount of service time. Efficiency requires to minimize the total costs incurred by the agents. On the other hand, fairness requires that agents served earlier should give compensations to agents served later. We are interested in finding the order in which to serve agents and the (positive or negative) monetary compensations they should receive. Each agent's utility is equal to his monetary compensation minus his

R. van den Brink $(\varangle)$

Department of Econometrics and Tinbergen Institute, VU University,

De Boelelaan 1105, 1081 HV Amsterdam, The Netherlands

e-mail: jrbrink@feweb.vu.nl

Y. Chun

Department of Economics, Seoul National University, Seoul 151-746, Korea

e-mail: ychun@snu.ac.kr 
total waiting cost. This sequencing problem has been studied extensively in the recent literature: from the incentive viewpoint (Dolan 1978; Suijs 1996; Mitra 2001, 2002) and from the normative viewpoint (Maniquet 2003; Chun 2011, 2006a,b; Mishra and Rangarajan 2007; Moulin 2007). Two well-known subclasses of sequencing problems are (i) a queueing problem in which all agents are assumed to need the same amount of service time but they differ in their unit waiting cost, and (ii) a scheduling problem in which all agents are assumed to have the same unit waiting cost but need (possibly) different amount of service times.

This article focuses on the normative approach. Two solutions or rules that have played important roles in the normative approach are the minimal and the maximal transfer rules. As shown in Maniquet (2003) and Chun (2006a) for queueing problems, and their generalization to sequencing problems by Chun (2011), these two rules can be obtained by applying the Shapley value (Shapley 1953) to corresponding cooperative games in which the worth of a coalition is appropriately defined from the sequencing problem. For the minimal transfer rule, the worth of a coalition is defined to be the minimum waiting cost incurred by its members under the assumption that they are served before the non-coalitional members. For the maximal transfer rule, it is defined to be the minimum waiting cost incurred by its members under the assumption that they are served after the non-coalitional members. ${ }^{1}$

In this article, we investigate how the minimal and the maximal transfer rules respond to changes in the set of agents. Our first main axiom is balanced consistency, which requires that the effect on the payoff from the departure of one agent to another agent should be equal between any two agents. We show that the minimal transfer rule is the only rule satisfying efficiency, Pareto indifference, and balanced consistency. On the other hand, the maximal transfer rule can be characterized by an alternative formulation of balanced consistency under constant completion time: upon the departure of an agent, all of his predecessors are assumed to move back by one position to keep the completion time constant. Under this alternative formulation, the maximal transfer rule becomes the only rule satisfying efficiency, Pareto indifference, and balanced consistency under constant completion time.

Our second main axiom is balanced cost reduction, which requires that if one agent leaves a problem, then the total payoffs of the remaining agents should be affected by the amount previously assigned to the leaving agent. Once again, the minimal transfer rule is the only rule satisfying efficiency, Pareto indifference, and balanced cost reduction.

This article is organized as follows. Section 2 contains some preliminaries and introduces rules. Section 3 explores the implications of balanced consistency and presents our first characterization of the minimal transfer rule. Section 4 explores the

\footnotetext{
1 This is different from the sequencing games with an initial order of Curiel et al. (1989), where agents (or jobs) are ordered in an initial queue. The question then is how agents should compensate one another when re-ordering into an efficient queue, taking account of the initial order. The sequencing games with an initial order are line-graph games, where only coalitions of consecutive agents (in the initial order) can have non-zero dividend (see van den Brink et al. 2007), while sequencing games as referred to in this article are two-games (i.e. only coalitions of size 2 can have a non-zero dividend, see Harsanyi (1959)).
} 
implications of balanced cost reduction and presents our second characterization of the minimal transfer rule. Concluding remarks follow in Sect. 5.

\section{Preliminaries}

Let $I \equiv\{1,2, \ldots\}$ be a (finite or infinite) universe of "potential" agents, and $\mathcal{N}$ the family of non-empty subsets of $I$. Each agent $i \in I$ is characterized by his service time, $r_{i}>0$, and his unit waiting cost, $\theta_{i} \geq 0$. A sequencing problem is defined as a list $(N, r, \theta)$ where $N \in \mathcal{N}$ is the set of agents, $r=\left(r_{i}\right)_{i \in N} \in \mathbb{R}_{++}^{N}$ is the vector of service times, and $\theta=\left(\theta_{i}\right)_{i \in N} \in \mathbb{R}_{+}^{N}$ is the vector of unit waiting costs. Let $\mathcal{S}^{N}$ be the class of all sequencing problems for $N$ and $\mathcal{S}=\bigcup_{N \in \mathcal{N}} \mathcal{S}^{N}$. Two subclasses of sequencing problems introduced earlier are a quеиеing problem, where for each $i, j \in I, r_{i}=r_{j}$, and a scheduling problem, where for each $i, j \in I, \theta_{i}=\theta_{j}$.

An allocation for $(N, r, \theta) \in \mathcal{S}^{N}$ is a pair $(\sigma, t) \in\{1, \ldots,|N|\}^{N} \times \mathbb{R}^{N}$, where for each $i \in N, \sigma_{i}$ denotes agent $i$ 's position in the queue and $t_{i}$ the monetary transfer to him. Let $P_{i}(\sigma)=\left\{j \in N \mid \sigma_{j}<\sigma_{i}\right\}$ be the set of agents preceding agent $i$ in $\sigma$, and $F_{i}(\sigma)=\left\{j \in N \mid \sigma_{j}>\sigma_{i}\right\}$ the set of agents following him. The agent who is served first incurs no waiting cost. The waiting cost of agent $i \in N$ in the queue $\sigma$ is $\sum_{j \in P_{i}(\sigma)} r_{j} \theta_{i}$. We assume that each agent $i \in N$ has a quasi-linear utility function, so that his utility from consuming the bundle $(\sigma, t)$ is given by $u_{i}(\sigma, t)=$ $t_{i}-\sum_{j \in P_{i}(\sigma)} r_{j} \theta_{i}$, where $t_{i}$ is the transfer of agent $i$ and $-\sum_{j \in P_{i}(\sigma)} r_{j} \theta_{i}$ is the total waiting cost of agent $i$ in the queue $\sigma$. An allocation is feasible if no two agents are assigned the same position, and the sum of all the transfers is not positive. Thus, the set of feasible allocations $Z(N, r, \theta)$ consists of all pairs $z=(\sigma, t) \in\{1, \ldots,|N|\}^{N} \times \mathbb{R}^{N}$ such that for all $i, j \in N, i \neq j$ implies $\sigma_{i} \neq \sigma_{j}$ and $\sum_{i \in N} t_{i} \leq 0$.

Given $(N, r, \theta) \in \mathcal{S}^{N}$, an allocation $(\sigma, t) \in Z(N, r, \theta)$ is queue-efficient if it minimizes the total waiting cost among the feasible allocations, that is, for all $\left(\sigma^{\prime}, t^{\prime}\right) \in$ $Z(N, r, \theta), \sum_{i \in N} \sum_{j \in P_{i}(\sigma)} r_{j} \theta_{i} \leq \sum_{i \in N} \sum_{j \in P_{i}\left(\sigma_{i}^{\prime}\right)} r_{j} \theta_{i}$. As shown in Smith (1956), total waiting cost is minimized if the agents are served in nonincreasing order with respect to their urgency index $\theta_{i} / r_{i}$. For $i, j \in N$, if $\theta_{i} / r_{i}=\theta_{j} / r_{j}$, then agents $i$ and $j$ have equivalent urgency indexes. The efficient queues do not depend on the transfers. Moreover, it is unique except for agents with equivalent urgency indexes, who will be next to each other in the queue and can be permuted. The set of efficient queues for $(N, r, \theta) \in \mathcal{S}^{N}$ is denoted by $\operatorname{Eff}(N, r, \theta)$. An allocation $(\sigma, t) \in Z(N, r, \theta)$ is budget balanced if $\sum_{i \in N} t_{i}=0$. A feasible allocation is efficient if it is queue-efficient and budget balanced.

A rule is a mapping $\varphi: \mathcal{S} \rightarrow \bigcup_{N \in \mathcal{N}} Z(N, r, \theta)$, which associates with each problem $(N, r, \theta) \in \mathcal{S}$ a non-empty subset $\varphi(N, r, \theta) \subset Z(N, r, \theta)$ of feasible allocations. A pair $\left(\sigma_{i}, t_{i}\right) \in \varphi_{i}(N, r, \theta)$ represents the position $\sigma_{i}$ of $i$ in the queue and his transfer $t_{i}$ in $(N, r, \theta)$. If the monetary transfer of an agent is positive, then this agent receives a compensation from other agents. If it is negative, then he/she has to pay that amount as compensation to other agents.

We mention two standard axioms for rules. First, a rule should choose an efficient (i.e. queue-efficient and budget balanced) allocation. 
Efficiency: For all $N \in \mathcal{N}$, all $(N, r, \theta) \in \mathcal{S}^{N}$, and all $(\sigma, t) \in \varphi(N, r, \theta)$, we have $\sigma \in \operatorname{Eff}(N, r, \theta)$ and $\sum_{i \in N} t_{i}=0$.

Second, if an allocation is chosen by a rule, then all other allocations which assign the same utility to each agent should be chosen by the rule.

Pareto indifference: For all $N \in \mathcal{N}$, all $(N, r, \theta) \in \mathcal{S}^{N}$, all $(\sigma, t) \in \varphi(N, r, \theta)$, and $\left(\sigma^{\prime}, t^{\prime}\right) \in Z(N, r, \theta)$, if $u_{i}\left(\sigma^{\prime}, t^{\prime}\right)=u_{i}(\sigma, t)$ for all $i \in N$, then $\left(\sigma^{\prime}, t^{\prime}\right) \in \varphi(N, r, \theta)$.

Next we recall two rules studied in Maniquet (2003) and Chun (2006a) for queueing problems, and generalized to sequencing problems by Chun (2011). The minimal transfer rule selects an efficient queue and transfers from each agent a half of his waiting cost multiplied by the sum of all his predecessors' service times minus a half of the sum of the unit waiting cost over all his/her followers multiplied by his/her own service time.

Minimal transfer rule, $\varphi^{M}$ : For all $N \in \mathcal{N}$, and all $(N, r, \theta) \in \mathcal{S}$, $\varphi^{M}(N, r, \theta)=\left\{\begin{array}{l|c}\sigma^{M} \in \operatorname{Eff}(N, r, \theta) \text { and } \\ t_{i}^{M}=\sum_{j \in P_{i}\left(\sigma^{M}\right)} \frac{r_{j} \theta_{i}}{2}-\sum_{j \in F_{i}\left(\sigma^{M}\right)} \frac{r_{i} \theta_{j}}{2}\end{array}\right\}$.

On the other hand, the maximal transfer rule selects an efficient queue and transfers to each agent a half of the sum of the unit waiting cost over all his predecessors multiplied by his/her own service time minus a half of his/her waiting cost multiplied by the sum of each of his followers' service time.

Maximal transfer rule, $\varphi^{X}$ : For all $N \in \mathcal{N}$, and all $(N, r, \theta) \in \mathcal{S}$, $\varphi^{X}(N, r, \theta)=\left\{\left(\sigma^{X}, t^{X}\right) \in Z(N, r, \theta) \mid \begin{array}{c}\sigma^{X} \in \operatorname{Eff}(N, r, \theta) \text { and } \\ t_{i}^{X}=\sum_{j \in P_{i}\left(\sigma^{X}\right)} \frac{r_{i} \theta_{j}}{2}-\sum_{j \in F_{i}\left(\sigma^{X}\right)} \frac{r_{j} \theta_{i}}{2}\end{array}\right\}$.

Note that the minimal and the maximal transfer rules assign a unique allocation if and only if all agents have different urgency index $\theta_{i} / r_{i}$. However, even when some agents have the same urgency index, agents' utilities do not depend on the choice of efficient queues if the compensation is determined according to the minimal or the maximal transfer rule. Thus, both rules are essentially single-valued, in the sense that for a given problem, each agent's utility is the same at all allocations that the rule chooses. As a consequence, any efficient queue can be chosen to calculate the utilities assigned by the two rules. To be specific, for all $N \in \mathcal{N}$, and all $(N, r, \theta) \in \mathcal{S}^{N}$, for the minimal transfer rule, the utility of agent $i$ is given by

$$
u_{i}\left(\sigma^{M}, t^{M}\right)=-\sum_{j \in P_{i}\left(\sigma^{M}\right)} r_{j} \theta_{i}+t_{i}^{M}=-\sum_{j \in P_{i}\left(\sigma^{M}\right)} \frac{r_{j} \theta_{i}}{2}-\sum_{j \in F_{i}\left(\sigma^{M}\right)} \frac{r_{i} \theta_{j}}{2}
$$


and for the maximal transfer rule,

$$
\begin{aligned}
u_{i}\left(\sigma^{X}, t^{X}\right) & =-\sum_{j \in P_{i}\left(\sigma^{X}\right)} r_{j} \theta_{i}+t_{i}^{X}=-\sum_{j \in P_{i}\left(\sigma^{X}\right)} r_{j} \theta_{i}+\sum_{j \in P_{i}\left(\sigma^{X}\right)} \frac{r_{i} \theta_{j}}{2}-\sum_{j \in F_{i}\left(\sigma^{X}\right)} \frac{r_{j} \theta_{i}}{2} \\
& =-\sum_{j \in N \backslash\{i\}} r_{j} \theta_{i}+\sum_{j \in P_{i}\left(\sigma^{X}\right)} \frac{r_{i} \theta_{j}}{2}+\sum_{j \in F_{i}\left(\sigma^{X}\right)} \frac{r_{j} \theta_{i}}{2}
\end{aligned}
$$

As mentioned in the introduction, the minimal and the maximal transfer rules can be obtained by applying the Shapley value (1953) to corresponding TU-games in which the worth of a coalition is appropriately defined. For the minimal transfer rule, the worth of a coalition is defined to be the minimum waiting cost incurred by its members under the assumption that they are served before the non-coalitional members. For the maximal transfer rule, it is defined to be the minimum waiting cost incurred by its members under the assumption that they are served after the non-coalitional members.

\section{Balanced consistency in sequencing problems}

If an agent leaves a sequencing problem, then it will affect the payoffs of other remaining agents. Balanced consistency requires that the effect of agent $i$ leaving the sequencing problem on the payoff of another agent $j \neq i$ should be the same as the effect of agent $j$ leaving the sequencing problem on the payoff of agent $i$. It is similar to "preservation of differences' of solutions for TU-games as discussed in Hart and Mas-Colell (1989). ${ }^{2}$ To stress the fact that our axiom concerns situations in which an agent leaves the sequencing problem similar as players leave a game in (reduced game) consistency properties, we refer to this property as balanced consistency. ${ }^{3}$

For all $(N, r, \theta) \in \mathcal{S}^{N}$ and all $j \in N$, let $r^{-j} \in \mathbb{R}_{++}^{N \backslash\{j\}}$ and $\theta^{-j} \in \mathbb{R}_{+}^{N \backslash\{j\}}$ be the projections given by $r^{-j}=\left(r_{k}\right)_{k \in N \backslash\{j\}}$ and $\theta^{-j}=\left(\theta_{k}\right)_{k \in N \backslash\{j\}}$.

Balanced consistency: For all $N \in \mathcal{N}$, all $(N, r, \theta) \in \mathcal{S}$, all $i, j \in N$, all $(\sigma, t) \in \varphi(N, r, \theta)$, all $\left(\sigma^{-i}, t^{-i}\right) \in \varphi\left(N \backslash\{i\}, r^{-i}, \theta^{-i}\right)$, and all $\left(\sigma^{-j}, t^{-j}\right) \in$ $\varphi\left(N \backslash\{j\}, r^{-j}, \theta^{-j}\right)$,

$$
u_{i}(\sigma, t)-u_{i}\left(\sigma^{-j}, t^{-j}\right)=u_{j}(\sigma, t)-u_{j}\left(\sigma^{-i}, t^{-i}\right)
$$

Now we investigate the implications of balanced consistency in the context of sequencing problems. First, we show that the minimal transfer rule satisfies the property.

\footnotetext{
2 This property states that the effect of player $i$ leaving the game on the payoff of player $j \neq i$ is equal to the effect of player $j$ leaving the game on the payoff of player $i$.

${ }^{3}$ Note that in the balanced contributions property as introduced by Myerson (1980) for cooperative games with a restricted set of feasible coalitions, the player set is fixed.
} 
Lemma 1 The minimal transfer rule satisfies balanced consistency.

Proof Let $(N, r, \theta) \in \mathcal{S}$. From the essential single-valuedness of $\varphi^{M}$, we may choose any $(\sigma, t) \in \varphi^{M}(N, r, \theta)$. Let $i, j \in N$ be such that $j \in P_{i}(\sigma)$ (and thus $\left.i \in F_{j}(\sigma)\right)$. To simplify the notation, we do not attach the superscript $M$ to $\sigma$ and $t$. Then, for all $\left(\sigma^{-i}, t^{-i}\right) \in \varphi^{M}\left(N \backslash\{i\}, r^{-i}, \theta^{-i}\right)$, and all $\left(\sigma^{-j}, t^{-j}\right) \in \varphi^{M}\left(N \backslash\{j\}, r^{-j}, \theta^{-j}\right)$,

$$
\begin{aligned}
& u_{i}(\sigma, t)-u_{i}\left(\sigma^{-j}, t^{-j}\right) \\
& =-\sum_{k \in P_{i}(\sigma)} \frac{r_{k} \theta_{i}}{2}-\sum_{k \in F_{i}(\sigma)} \frac{r_{i} \theta_{k}}{2}-\left(-\sum_{k \in P_{i}(\sigma) \backslash\{j\}} \frac{r_{k} \theta_{i}}{2}-\sum_{k \in F_{i}(\sigma)} \frac{r_{i} \theta_{k}}{2}\right) \\
& =-\frac{r_{j} \theta_{i}}{2}
\end{aligned}
$$

and

$$
\begin{aligned}
& u_{j}(\sigma, t)-u_{j}\left(\sigma^{-i}, t^{-i}\right) \\
& =-\sum_{k \in P_{j}(\sigma)} \frac{r_{k} \theta_{j}}{2}-\sum_{k \in F_{j}(\sigma)} \frac{r_{j} \theta_{k}}{2}-\left(-\sum_{k \in P_{j}(\sigma)} \frac{r_{k} \theta_{j}}{2}-\sum_{k \in F_{j}(\sigma) \backslash\{i\}} \frac{r_{j} \theta_{k}}{2}\right) \\
& =-\frac{r_{j} \theta_{i}}{2} .
\end{aligned}
$$

Altogether, we conclude that the minimal transfer rule satisfies balanced consistency.

We ask whether there is any other rule satisfying efficiency and Pareto indifference together with balanced consistency. As it turns out, the minimal transfer rule is the only one satisfying the three axioms together. Before preceding to our first main result, we state the following lemma.

Lemma 2 If a rule $\varphi$ is efficient, then for all $(N, r, \theta) \in \mathcal{S}$, all $(\sigma, t) \in \varphi(N, r, \theta)$, all $i \in N$, and all $\left(\sigma^{-i}, t^{-i}\right) \in \varphi\left(N \backslash\{i\}, r^{-i}, \theta^{-i}\right)$,

$$
\sum_{j \in N} u_{j}(\sigma, t)-\sum_{j \in N \backslash\{i\}} u_{j}\left(\sigma^{-i}, t^{-i}\right)=-\sum_{k \in P_{i}(\sigma)} r_{k} \theta_{i}-\sum_{k \in F_{i}(\sigma)} r_{i} \theta_{k} .
$$

Proof For all $(\sigma, t) \in \varphi(N, r, \theta)$, all $i \in N$, and all $\left(\sigma^{-i}, t^{-i}\right) \in \varphi\left(N \backslash\{i\}, r^{-i}, \theta^{-i}\right)$, efficiency implies that

$$
\sum_{j \in N} u_{j}(\sigma, t)=-\sum_{j \in N} \sum_{k \in P_{j}(\sigma)} r_{k} \theta_{j}
$$

and

$$
\sum_{j \in N \backslash\{i\}} u_{j}\left(\sigma^{-i}, t^{-i}\right)=-\sum_{j \in P_{i}(\sigma)} \sum_{k \in P_{j}(\sigma)} r_{k} \theta_{j}-\sum_{j \in F_{i}(\sigma)} \sum_{k \in P_{j}(\sigma) \backslash\{i\}} r_{k} \theta_{j} .
$$


Since, by efficiency all agents are served in nonincreasing order with respect to their urgency indices, subtracting these two equations from each other, we obtain (4).

Now we are ready to show our first characterization result. We note that if $\sigma_{i} \in$ $\{1, \ldots,|N|\}$ is determined and $u_{i}(\sigma, t)$ is known, then also $t_{i}$ is determined.

Theorem 1 The minimal transfer rule is the only rule satisfying efficiency, Pareto indifference, and balanced consistency.

Proof It is well known that the minimal transfer rule satisfies efficiency and Pareto indifference, and from Lemma 1, it satisfies balanced consistency. Conversely, let $\varphi$ be a rule satisfying the three axioms. Let $N \in \mathcal{N}$ and $(N, r, \theta) \in \mathcal{S}^{N}$ be given. If $|N|=1$, then efficiency implies that $\sigma_{i}=1$ and $t_{i}=0$ for $i \in N$.

Let $N$ be such that $|N|=2$. Without loss of generality, we may assume that $N \equiv\{i, j\}$ and that $\theta_{i} / r_{i} \geq \theta_{j} / r_{j}$. Let $(\sigma, t) \in \varphi(N, r, \theta),\left(\sigma^{-i}, t^{-i}\right) \in$ $\varphi\left(N \backslash\{i\}, r^{-i}, \theta^{-i}\right)$, and $\left(\sigma^{-j}, t^{-j}\right) \in \varphi\left(N \backslash\{j\}, r^{-j}, \theta^{-j}\right)$. By balanced consistency, $u_{i}(\sigma, t)-u_{i}\left(\sigma^{-j}, t^{-j}\right)=u_{j}(\sigma, t)-u_{j}\left(\sigma^{-i}, t^{-i}\right)$. Since $u_{i}\left(\sigma^{-j}, t^{-j}\right)=$ $u_{j}\left(\sigma^{-i}, t^{-i}\right)=0$, we have $u_{i}(\sigma, t)=u_{j}(\sigma, t)$. By efficiency, $u_{i}(\sigma, t)+u_{j}(\sigma, t)=$ $-r_{i} \theta_{j}$. Altogether, we obtain $u_{i}(\sigma, t)=u_{j}(\sigma, t)=-\frac{r_{i} \theta_{j}}{2}$. By efficiency and Pareto indifference, we may assume that $\sigma_{i}=1$ and $\sigma_{j}=2$. Then, $t_{i}=-\frac{r_{i} \theta_{j}}{2}=-t_{j}$, as desired.

We will establish the claim for an arbitrary number of agents by an induction argument. As induction hypothesis, suppose that $\varphi\left(N^{\prime}, r^{\prime}, \theta^{\prime}\right)=\varphi^{M}\left(N^{\prime}, r^{\prime}, \theta^{\prime}\right)$ whenever $\left|N^{\prime}\right| \leq|N|-1$. Let $N \equiv\{1,2, \ldots, n\}$, and let $(\sigma, t) \in \varphi(N, r, \theta)$. By efficiency and Pareto indifference, we may assume without loss of generality that $\theta_{1} / r_{1} \geq \theta_{2} / r_{2} \geq$ $\cdots \geq \theta_{n} / r_{n}$, and that $\sigma_{i}=i$ for all $i \in N$. For any $i \in N$, let $\left(\sigma^{-i}, t^{-i}\right) \in$ $\varphi\left(N \backslash\{i\}, r^{-i}, \theta^{-i}\right)$. By balanced consistency, $u_{i}(\sigma, t)-u_{i}\left(\sigma^{-j}, t^{-j}\right)=u_{j}(\sigma, t)-$ $u_{j}\left(\sigma^{-i}, t^{-i}\right)$ for any $i, j \in N$. Now fix $i$, change $j \neq i$ from 1 to $n$, and add up the $(n-1)$ equations obtained in this way. We have:

$$
(n-1) u_{i}(\sigma, t)-\sum_{j \in N \backslash\{i\}} u_{i}\left(\sigma^{-j}, t^{-j}\right)=\sum_{j \in N \backslash\{i\}}\left(u_{j}(\sigma, t)-u_{j}\left(\sigma^{-i}, t^{-i}\right)\right) .
$$

Adding $u_{i}(\sigma, t)+\sum_{j \in N \backslash\{i\}} u_{i}\left(\sigma^{-j}, t^{-j}\right)$ to both sides gives

$$
n u_{i}(\sigma, t)=\sum_{j \in N} u_{j}(\sigma, t)-\sum_{j \in N \backslash\{i\}} u_{j}\left(\sigma^{-i}, t^{-i}\right)+\sum_{j \in N \backslash\{i\}} u_{i}\left(\sigma^{-j}, t^{-j}\right) .
$$

From the induction hypothesis, it follows that

$$
\begin{aligned}
\sum_{j \in N \backslash\{i\}} u_{i}\left(\sigma^{-j}, t^{-j}\right)= & -\sum_{j \in P_{i}(\sigma)}\left(\sum_{k \in P_{i}(\sigma) \backslash\{j\}} \frac{r_{k} \theta_{i}}{2}-\sum_{k \in F_{i}(\sigma)} \frac{r_{i} \theta_{k}}{2}\right) \\
& +\sum_{j \in F_{i}(\sigma)}\left(\sum_{k \in P_{i}(\sigma)} \frac{r_{k} \theta_{i}}{2}-\sum_{k \in F_{i}(\sigma) \backslash\{j\}} \frac{r_{i} \theta_{k}}{2}\right)
\end{aligned}
$$




$$
\begin{aligned}
= & -(i-2) \sum_{k \in P_{i}(\sigma)} \frac{r_{k} \theta_{i}}{2}-(i-1) \sum_{k \in F_{i}(\sigma)} \frac{r_{i} \theta_{k}}{2} \\
& -(n-i) \sum_{k \in P_{i}(\sigma)} \frac{r_{k} \theta_{i}}{2}-(n-i-1) \sum_{k \in F_{i}(\sigma)} \frac{r_{i} \theta_{k}}{2} \\
= & -(n-2) \sum_{k \in P_{i}(\sigma)} \frac{r_{k} \theta_{i}}{2}-(n-2) \sum_{k \in F_{i}(\sigma)} \frac{r_{i} \theta_{k}}{2} .
\end{aligned}
$$

Using Lemma 2, substituting (4) and (6) into (5) yields

$$
\begin{aligned}
n u_{i}(\sigma, t)= & -2 \sum_{k \in P_{i}(\sigma)} \frac{r_{k} \theta_{i}}{2}-2 \sum_{k \in F_{i}(\sigma)} \frac{r_{i} \theta_{k}}{2} \\
& -(n-2) \sum_{k \in P_{i}(\sigma)} \frac{r_{k} \theta_{i}}{2}-(n-2) \sum_{k \in F_{i}(\sigma)} \frac{r_{i} \theta_{k}}{2} \\
= & -n \sum_{k \in P_{i}(\sigma)} \frac{r_{k} \theta_{i}}{2}-n \sum_{k \in F_{i}(\sigma)} \frac{r_{i} \theta_{k}}{2},
\end{aligned}
$$

or equivalently,

$$
u_{i}(\sigma, t)=-\sum_{k \in P_{i}(\sigma)} \frac{r_{k} \theta_{i}}{2}-\sum_{k \in F_{i}(\sigma)} \frac{r_{i} \theta_{k}}{2}=u_{i}\left(\sigma^{M}, t^{M}\right) .
$$

By efficiency, $\sigma \in \operatorname{Eff}(N, r, \theta)$, and thus (7) fixes the transfers

$$
t_{i}=\sum_{j \in P_{i}(\sigma)} \frac{r_{j} \theta_{i}}{2}-\sum_{j \in F_{i}(\sigma)} \frac{r_{i} \theta_{j}}{2}=t_{i}^{M},
$$

as desired.

Remark 1 Although 'preservation of differences' is typical for the Shapley value, it is not obvious that balanced consistency for sequencing problems characterizes the minimal transfer rule since the maximal transfer rule is also obtained as the Shapley value of an associated TU-game.

Remark 2 Upon the departure of an agent, if we assume that all of his/her predecessors are moving back by one position to keep the same completion time, an alternative balanced consistency under constant completion time property can be formulated. The maximal transfer rule is the only rule satisfying efficiency, Pareto indifference, and balanced consistency under constant completion time.

\section{Balanced cost reduction in sequencing problems}

Suppose that an agent leaves a sequencing problem. Since the agent is not in the queue anymore, the total waiting cost of all the remaining agents will be decreased. In other 
words, the presence of an agent generates a negative externality to any other agent. Balanced cost reduction requires that the total (over all remaining agents) decrease in this negative externality as a result of the departure of an agent be equal to the negative of the payoff of the departing agent when he is still present.

Balanced cost reduction: For all $N \in \mathcal{N}$, all $(N, r, \theta) \in \mathcal{S}$, all $j \in N$, all $(\sigma, t) \in$ $\varphi(N, r, \theta)$, and all $\left(\sigma^{-j}, t^{-j}\right) \in \varphi\left(N \backslash\{j\}, r^{-j}, \theta^{-j}\right)$,

$$
\sum_{i \in N \backslash\{j\}}\left(u_{i}(\sigma, t)-u_{i}\left(\sigma^{-j}, t^{-j}\right)\right)=u_{j}(\sigma, t) .
$$

We explore the implications of balanced cost reduction in the context of sequencing problems. First, we show that the minimal transfer rule satisfies the property.

Lemma 3 The minimal transfer rule satisfies balanced cost reduction.

Proof Let $(N, r, \theta) \in \mathcal{S}$ and $j \in N$. From the essential single-valuedness of $\varphi^{M}$, we may choose any $(\sigma, t) \in \varphi^{M}(N, r, \theta)$ and any $\left(\sigma^{-j}, t^{-j}\right) \in \varphi^{M}\left(N \backslash\{j\}, r^{-j}, \theta^{-j}\right)$. To simplify the notation, we do not attach the superscript $M$ to $\sigma$ and $t$. From (2) and (3) in Lemma 1, we can derive that

$$
\begin{aligned}
\sum_{i \in N \backslash\{j\}}\left(u_{i}(\sigma, t)-u_{i}\left(\sigma^{-j}, t^{-j}\right)\right) & =-\sum_{i \in P_{j}(\sigma)} \frac{r_{i} \theta_{j}}{2}-\sum_{i \in F_{j}(\sigma)} \frac{r_{j} \theta_{i}}{2} \\
& =u_{j}(\sigma, t),
\end{aligned}
$$

showing that the minimal transfer rule satisfies balanced cost reduction.

Next we present our second characterization.

Theorem 2 The minimal transfer rule is the only rule satisfying efficiency, Pareto indifference, and balanced cost reduction.

Proof It is well known that the minimal transfer rule satisfies efficiency and Pareto indifference, and from Lemma 3, it satisfies balanced cost reduction.

Conversely, let $\varphi$ be a rule satisfying the three axioms. Let $N \in \mathcal{N}$ and $(N, r, \theta) \in$ $\mathcal{S}^{N}$ be given. If $|N|=1$, then efficiency implies that $\sigma_{i}=1$ and $t_{i}=0$ for $i \in N$. Now let $N$ be such that $|N| \geq 2, j \in N$ be a leaving agent, and $\left(\sigma^{-j}, t^{-j}\right) \in$ $\varphi\left(N \backslash\{j\}, r^{-j}, \theta^{-j}\right)$. By balanced cost reduction,

$$
\sum_{i \in N \backslash\{j\}}\left(u_{i}(\sigma, t)-u_{i}\left(\sigma^{-j}, t^{-j}\right)\right)=u_{j}(\sigma, t) .
$$

Adding $u_{j}(\sigma, t)$ to both sides gives

$$
\sum_{i \in N} u_{i}(\sigma, t)-\sum_{i \in N \backslash\{j\}} u_{i}\left(\sigma^{-j}, t^{-j}\right)=2 u_{j}(\sigma, t) .
$$


Using Lemma 2, and substituting (4) into (8) yields

$$
2 u_{j}(\sigma, t)=-\sum_{i \in P_{j}(\sigma)} r_{i} \theta_{j}-\sum_{i \in F_{j}(\sigma)} r_{j} \theta_{i}
$$

which implies that

$$
u_{j}(\sigma, t)=-\sum_{i \in P_{j}(\sigma)} \frac{r_{i} \theta_{j}}{2}-\sum_{i \in F_{j}(\sigma)} \frac{r_{j} \theta_{i}}{2}=u_{j}\left(\sigma^{M}, t^{M}\right) .
$$

By efficiency and Pareto indifference, we choose all efficient queues in $E f f(N, r, \theta)$, and for any $\sigma \in \operatorname{Eff}(N, r, \theta)$, (9) fixes the transfers

$$
t_{j}=\sum_{i \in P_{j}(\sigma)} \frac{r_{i} \theta_{j}}{2}-\sum_{i \in F_{j}(\sigma)} \frac{r_{j} \theta_{i}}{2}=t_{j}^{M}
$$

the desired expression. ${ }^{4}$

\section{Concluding remarks}

In this article, we presented two axiomatic characterizations of the minimal transfer rule in the context of sequencing problems on the basis of balanced consistency or balanced cost reduction in addition to efficiency and Pareto indifference. We note that all our results carry over to two subclasses of sequencing problems: queueing problems and scheduling problems.

Another axiom widely discussed in the literature specifying how a rule should respond to changes in the population is population solidarity (Thomson 1983; Chun 1986, and others ${ }^{5}$ ): it requires that upon the departure of an agent, all the remaining agents should be affected in the same direction, all gain or all lose. As discussed in Chun (2006a) for queueing problems, the minimal transfer rule for sequencing problems also satisfies population solidarity, but the maximal transfer rule does not satisfy it. On the other hand, as in Remark 1, upon the departure of an agent, if we assume that all of his predecessors are moving back by one position to keep the completion time constant, then both the minimal and the maximal transfer rules satisfy the alternative population solidarity under constant completion time property. It remains an open question whether the minimal or the maximal transfer rules can be characterized on the basis of population solidarity.

Another question for future research is to investigate axioms concerning changes in the parameters of the sequencing problem without changing the set of agents, such as the before mentioned balanced contributions property (Myerson 1980), fairness

\footnotetext{
${ }^{4}$ Note the difference with the end of the proof of Theorem 1. In the proof of Theorem 2, we need to make sure to choose all efficient queues, and for any efficient queue, we have the desired formula. In the induction proof of Theorem 1, one efficient queue is chosen already from the beginning.

${ }^{5}$ See Thomson (1995) for a survey.
} 
(Myerson 1977; van den Brink 2001) or monotonicity (Young 1985; van den Brink 2007).

Acknowledgments The authors are grateful to Kyung Hwan Baik, Francois Maniquet, and two referees for their comments. Chun's work was supported by the National Research Foundation of Korea Grant funded by the Korean Government (KRF-2009-342-B00011) and the Netherlands Organization for Scientific Research (NWO) grant 040.11.143.

Open Access This article is distributed under the terms of the Creative Commons Attribution Noncommercial License which permits any noncommercial use, distribution, and reproduction in any medium, provided the original author(s) and source are credited.

\section{References}

Chun Y (1986) The solidarity axiom for quasi-linear social choice problems. Soc Choice Welf 3:297-310 Chun Y (2011) Consistency and monotonicity in sequencing problems. Int J Game Theory 40:29-41

Chun Y (2006) A pessimistic approach to the queueing problem. Math Soc Sci 51:171-181

Chun Y (2006) No-envy in queueing problems. Econ Theory 29:151-162

Curiel I, Pederzoli G, Tijs S (1989) Sequencing games. Eur J Oper Res 40:344-351

Dolan R (1978) Incentive mechanisms for priority queueing problems. Bell J Econ 9:421-436

Harsanyi JC (1959) A bargaining model for cooperative n-person games. In: Tucker AW, Luce RD (eds) Contributions to the theory of games IV. Princeton UP, Princeton pp 325-355

Hart S, Mas-Colell A (1989) Potential, value and consistency. Econometrica 57: 589-614

Maniquet F (2003) A characterization of the Shapley value in queueing problems. J Econ Theory 109: $90-103$

Mishra D, Rangarajan B (2007) Cost sharing in a job scheduling problem. Soc Choice Welf 29:369-382

Mitra M (2001) Mechanism design in queueing problems. Econ Theory 17:277-305

Mitra M (2002) Achieving the first best in sequencing problems. Rev Econ Des 7:75-91

Moulin H (2007) On scheduling fees to prevent merging, splitting, and transferring of jobs. Math Oper Res $32: 266-283$

Myerson RB (1977) Graphs and cooperation in games. Math Oper Res 2:225-229

Myerson RB (1980) Conference structures and fair allocation rules. Int J Game Theory 9:169-182

Shapley LS (1953) A value for n-person games. In: Kuhn HW, Tucker AW (eds) Contributions to the theory of games II, Annals of mathematics studies No 28. Princeton University Press, Princeton, NJ pp $307-317$

Smith W (1956) Various optimizers for single-stage production. Nav Res Logist Q 3:59-66

Suijs J (1996) On incentive compatibility and budget balancedness in public decision making. Econ Des 2:193-209

Thomson W (1983) Problems of fair division and the egalitarian solution. J Econ Theory 31:211-226

Thomson W (1995) Population monotonic allocation rules. In: Barnett WA, Moulin H, Salles M, Schofield NJ (eds) Social choice, welfare, and ethics. Cambridge University Press, Cambridge pp $79-124$

van den Brink R (2001) An axiomatization of the Shapley value using a fairness property. Int J Game Theory 30:309-319

van den Brink R (2007) Null or nullifying players: the difference between the Shapley value and equal division solutions. J Econ Theory 136:767-775

van den Brink R, van der Laan G, Vasil'ev VA (2007) Component efficient solutions in line-graph games with applications. Econ Theory 33:349-364

Young HP (1985) Monotonic solutions of cooperative games. Int J Game Theory 14:65-72 